\title{
MODELAGEM DA ALTURA DE Pinus caribaea var. hondurensis EM DIFERENTES ESPAÇAMENTOS
}

\author{
MODELING HEIGHT OF Pinus caribaea \\ var. hondurensis IN DIFFERENT SPACING
}

scientific journal

Recebido em 25/10/2017

Clebson Lima Cerqueira ${ }^{1}$

Aceito em 28/11/2017

Rômulo Môra

Publicado em 30/11/2017

Italo Luiz Corrêa Lenzi ${ }^{3}$

Ronaldo Drescher ${ }^{4}$

Edberto Moura Lima ${ }^{5}$

DOI: dx.doi.org/10.5380/biofix.v2i2.56012

Gerson Dos Santos Lisboa ${ }^{6}$

Luciano Cavalcante De Jesus França ${ }^{7}$

Universidade Federal de Mato Grosso, Cuiabá, Mato Grosso, Brasil
clebsonlima10@hotmail.com ${ }^{1}$,romulomef@yahoo.com.br ${ }^{2}$, italo_lenzi@hotmail.com ${ }^{3}$ \& ronaldodrescher@gmail.com ${ }^{4}$

Universidade Federal do Paraná, Curitiba, Paraná, Brasil edberto.moura@gmail.com ${ }^{5}$

Universidade Federal do Sul da Bahia, Itabuna, Bahia, Brasil gerson.lisboa@gmail.com ${ }^{6}$

Universidade Federal dos Vales do Jequitinhonha e Mucurí, Diamantina, Minas Gerais, Brasil lucianodejesus@florestal.eng.br ${ }^{7}$

\section{RESUMO}

Apesar do Pinus ser um gênero nativo do hemisfério norte, sua introdução nas regiões equatoriais ocorre gradativamente desde as últimas décadas, demonstrando sua capacidade de adaptabilidade e rusticidade. Devido sua importância comercial, se faz necessário o estudo de modelos para estimativa de variáveis dendrométricas. Dessa forma, este trabalho teve como objetivo ajustar e avaliar modelos tradicionais de relação hipsométrica e verificar o efeito de dois espaçamentos $(2,5 \mathrm{~m} \mathrm{x}$ $2,5 \mathrm{~m}$ e $2,0 \mathrm{~m} \times 2,5 \mathrm{~m}$ ) no ajuste das equações sobre um povoamento de Pinus caribaea var. hondurensis no município de Vilhena, Rondônia. A partir das variáveis dendrométricas de 405 árvores mensuradas, ajustou-se seis modelos estatísticos lineares e não lineares de relação hipsométricas. $\mathrm{N}$ avaliação dos modelos, analisou-se a influência do espaçamento na relação hipsométrica por meio do teste de identidade de modelos. As equações ajustadas apresentaram coeficiente de determinação ajustado de 0,32 a 0,57 e erro padrão médio em percentagem de $10,3 \%$ a $14,2 \%$. As análises gráficas demonstraram que o modelo logístico proporciona estimativas mais acuradas para predição da altura total nos diferentes tratamentos, ao passo que o teste de identidade de modelos realizados nos tratamentos agrupados apresentou resultados significativos, não sendo recomendado o ajuste de um modelo global para todo o conjunto de dados.

PALAVRAS-CHAVE: Inventário florestal, Modelagem florestal, Relação hipsométrica, Teste de identidade.

\section{ABSTRACT}

Despite of Pinus be a native genus of northern hemisphere, it has been gradually introduced into the equatorial regions in the last decades, demonstrating your capacity of adaptability and rusticity. Moreover, due your commercial importance, it makes necessary to study traditional models to estimate tree variables. In this way, this work aims to fit and evaluate traditional height-diameter models and verify the effect of two spacings $(2.5 \mathrm{~m} \times 2.5 \mathrm{~m}$ and $2.0 \mathrm{~m} \times 2.5$ $\mathrm{m})$ for fitting equations on a Pinus caribaea var. hondurensis stand in Vilhena, Rondônia. By means of tree variables of 405 measured trees, six linear and nonlinear height-diameter models were fitted. For evaluating models, the influence of spacings on the heightdiameter relationship was analyzed through identity test. The fitted equations showed adjusted coefficient of determination of 0.32 to 0.57 and standard error of the estimates of $10.3 \%$ to $14.2 \%$. The graph analyzes showed that the logistic model provides more accurate estimates for total height prediction in the different treatments, and the identity test performed in grouped treatments presented significant results, being not recommended to fit only one global model for the entire data set.

KEYWORDS: Forest inventory, Forest modeling, Height-diameter relationship, Identity test. 


\section{INTRODUÇÃO}

O setor silvicultural brasileiro tem contribuído de forma significativa para o suprimento da demanda por produtos de origem florestal, tornando-se, nos últimos anos, um dos mais relevantes no cenário global. Segundo IBÁ (2017), no ano de 2016, o Brasil apresentou 7,8 milhões de hectares ocupados por florestas plantadas, principalmente por espécies pertencentes aos gêneros Eucalyptus e Pinus.

Os povoamentos de Pinus estão distribuídos nas regiões Centro-Oeste, Sudeste e Sul do Brasil, ocupando aproximadamente 1,6 milhão de hectares. Contudo, a expansão e a viabilidade desses povoamentos em todas as regiões do Brasil somente foi possível após a introdução de diversas espécies do gênero, principalmente das regiões tropicais, constituindo uma importante fonte de matéria prima para diversos fins, tais como celulose, lâminas e chapas, madeira serrada, compensados, energia e resinagem (SHIMIZU e MEDRADO, 2006; DONADONI et al., 2010).

O Pinus caribaea Morelet é uma das espécies de Pinus mais cultivadas no país, a qual engloba três variedades (bahamensis, caribaea e hondurensis), dentre as quais o hondurensis, originário da América Central, é uma das mais cultivadas no mundo, devido sua ampla adaptação as diversas condições edafoclimáticas (MELO et al., 2013).

Com a expansão dos projetos florestais, é fundamental quantificar e prognosticar com precisão os recursos florestais. Dessa forma, os gestores têm buscado utilizar ferramentas e técnicas de modelagem para auxiliar no manejo dos povoamentos florestais. Dentre as várias técnicas utilizadas, tem-se os modelos hipsométricos para estimativa das alturas, que contribuem para a redução do tempo e dos custos nos inventários florestais.

O emprego da modelagem para estimativa de variáveis dendrométricas em povoamentos de Pinus estabelecidos na região Sul é um assunto bastante difundido. Entretanto, ainda são escassos os estudos com Pinus tropicais e na região Norte do Brasil, destacando-se os trabalhos de Donadoni et al. (2010) e Pelissari et al. (2011).

A relação hipsométrica sofre influência de diversos fatores ambientais e do povoamento, tais como: sítio, idade, material genético, densidade, tamanho da copa, arranjo espacial e tratamentos silviculturais. Dessa maneira, recomendam-se o ajuste de modelos para cada condição específica (CURTIS, 1967; SCOLFORO, 2005). Entretanto, Bartoszeck et al. (2002) acrescentam que os modelos hipsométricos que consideram esses fatores são empregados com menos frequência, sendo preferível o uso de equações tradicionais disponíveis na literatura.

Segundo Vendruscolo et al. (2015), em situações em que os povoamentos florestais são implantados ou conduzidos sob diferentes tratamentos, os ajustes de modelos hipsométricos devem ser realizados para cada situação, o que acarreta em número elevado de equações. Assim, faz-se necessário testar a existência da diferença estatística entre tratamentos por meio do teste de identidade de modelos, visando reduzir o número de equações.

Nesse contexto, considerando a escassez de estudos sobre a modelagem da altura de Pinus caribaea var. hondurensis (Sénécl.) W.H. Barrett \& Golfari, objetivou-se ajustar e avaliar modelos tradicionais de relação hipsométrica e verificar o efeito do espaçamento no ajuste das equações.

\section{MATERIAL E MÉTODOS}

\section{Caracterização da área de estudo}

Os dados utilizados neste estudo foram obtidos em povoamentos homogêneos de Pinus caribaea var. hondurensis, situados na fazenda Londrina, município de Vilhena, estado de Rondônia, Brasil (Figura 1). Segundo a classificação de Köppen, o clima da região é do tipo Aw (ALVARES et al., 2013), a precipitação média anual da região é de $2.068 \mathrm{~mm}$, com estação chuvosa entre os meses de outubro a abril e as temperaturas anuais máxima e mínima são de $29^{\circ} \mathrm{C}$ e $19,3^{\circ} \mathrm{C}$, respectivamente (RAMALHO et al., 2004).

\section{Base de dados}

Os dados para realização deste trabalho foram obtidos por meio de inventário florestal, em povoamentos de aproximadamente 8 anos de idade, implantados com mudas seminais. As variáveis dendrométricas mensuradas foram o diâmetro com casca a 1,30 $\mathrm{m}$ de altura $(d)$ com fita métrica e a altura total $(h)$ com auxílio do hipsômetro Haglöf. Esses dados foram obtidos em povoamentos com dois espaçamentos diferentes, perfazendo um total de 405 árvores mensuradas, conforme a Tabela 1.

Tabela 1. Descrição do povoamento estudado

\begin{tabular}{cccc}
\hline Tratamento & Espaçamento & Área (ha) & Árvores \\
\hline $\mathrm{T} 1$ & $2,5 \mathrm{~m} \times 2,5 \mathrm{~m}$ & 42 & 182 \\
$\mathrm{~T} 2$ & $2,0 \mathrm{~m} \times 2,5 \mathrm{~m}$ & 157 & 223 \\
\hline Total & & 199 & 405 \\
\hline
\end{tabular}




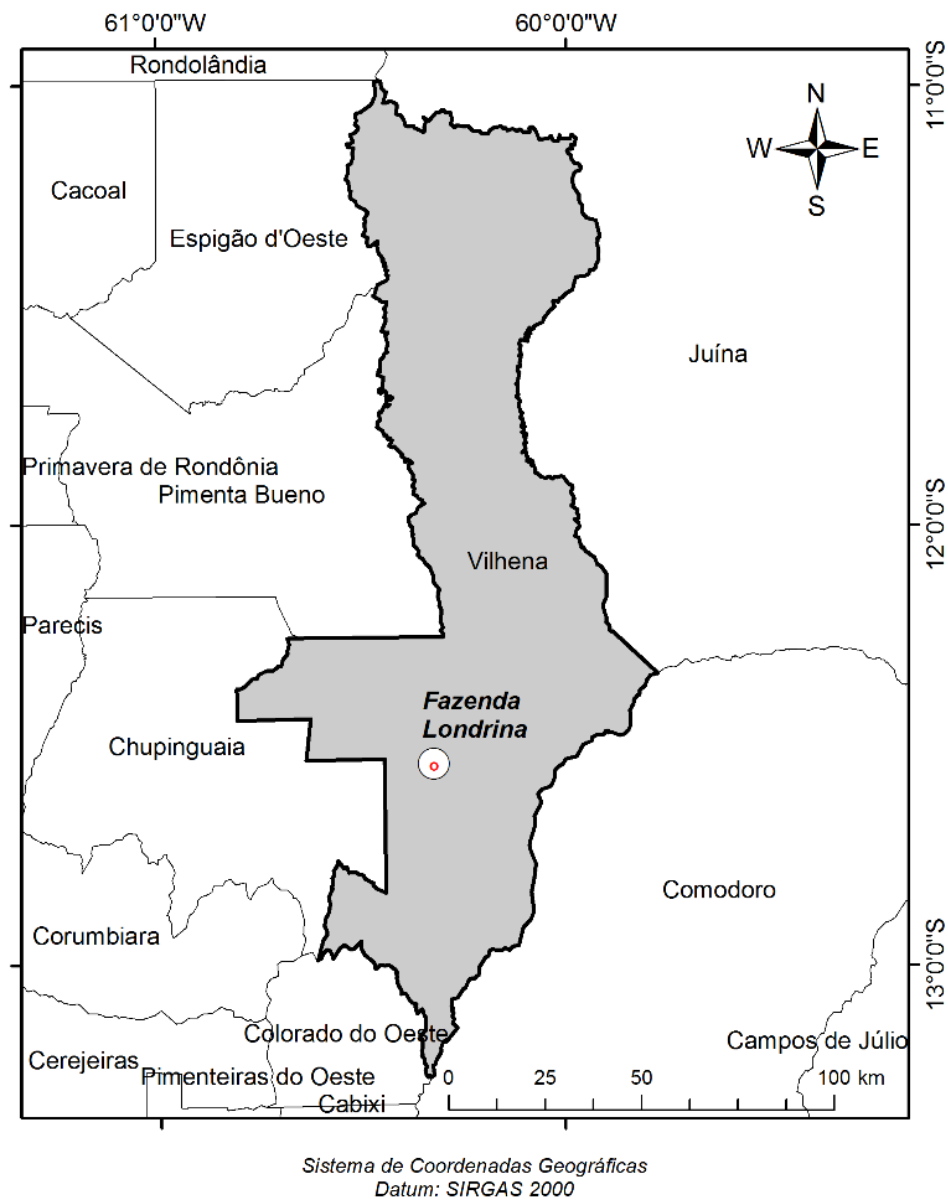

Figura 1. Localização da área de estudo em Vilhena, estado de Rondônia, Brasil.

A partir do banco de dados proveniente da amostragem e da mensuração das variáveis dendrométricas nos respectivos espaçamentos, foram ajustados e avaliados seis modelos estatísticos lineares e não lineares de relação hipsométricas (Tabela 2).

Tabela 2. Modelos de relação hipsométricas ajustados

\begin{tabular}{ccc}
\hline Número & Denominação & Modelo \\
\hline 1 & Stoffels & $\ln (h)=\beta_{0}+\beta_{1} \ln (d)+\varepsilon$ \\
2 & Curtis & $\ln (h)=\beta_{0}+\beta_{1} \frac{1}{d}+\varepsilon$ \\
3 & Hiperbólico & $h=\beta_{0}+\frac{\beta_{1}}{d^{2}}+\varepsilon$ \\
4 & Henriksen & $h=\beta_{0}+\beta_{1} \ln (h)+\varepsilon$ \\
5 & Assman & $h=\beta_{0}+\beta_{1} \frac{1}{d}+\varepsilon$ \\
6 & Logística & $h=\frac{\beta_{0}}{1+\beta_{1} e^{-\beta_{2} d}}$ \\
\hline
\end{tabular}

In = logaritmo natural; $h=$ altura total $(\mathrm{m}) ; d=$ diâmetro a $1,3 \mathrm{~m}$ de altura $(\mathrm{cm}) ; \beta_{i}=$ coeficiente de regressão; e $\varepsilon=$ erro aleatório.

A precisão e a acurácia dos modelos hipsométricos foram avaliados com base nos seguintes critérios estatísticos: coeficiente de determinação ajustado ( $R^{2} a j$.), erro padrão da estimativa em porcentagem (Syx\%), significância dos coeficientes de regressão $\left(\beta_{i}\right)$ e análise gráfica dos resíduos (E\%), calculados pelas Equações 1, 2 e 3.

$$
\begin{aligned}
& R_{a j .}^{2}=1-\left(\frac{S Q_{\text {res }}}{S Q_{\text {tot }}}\right) *\left(\frac{n-1}{n-p}\right) \\
& S_{y x}=\sqrt{\frac{\sum_{i=1}^{n}\left(Y_{i}-\hat{Y}_{i}\right)}{n-p}} \therefore S_{y x} \%=\frac{S_{y x}}{\bar{Y}} * 100 \\
& E_{\%}=\left(\frac{Y_{\mathrm{i}}-\hat{Y}_{\mathrm{i}}}{Y_{\mathrm{i}}}\right) * 100
\end{aligned}
$$

Em que: $Y_{i}=$ variável observada; $\hat{Y}_{i}=$ variável estimada; $\bar{Y}=$ média aritmética da variável; $n=$ número de observações; $p=$ número de coeficientes de regressão; $S Q_{\text {res }}=$ soma dos quadrados dos resíduos; e $S Q_{t o t}=$ soma de quadrados total.

Para a elaboração dos gráficos e a análise da homogeneidade dos resíduos, adotou-se o procedimento proposto por Mehtätalo (2008), em que linhas verticais são adicionadas para mostrar intervalos de confiança a 
95\% para observações individuais. Inicialmente, a função classifica os dados em classes, de forma que cada classe tenha aproximadamente o mesmo número de observações. Posteriormente, calcula-se a média e o desvio padrão ( $s$ ) em cada classe, sendo as linhas verticais plotadas. Quando a linha de comprimento 3,92*s não cruza o eixo $X$, a cor de realce vermelha é usada na linha indicando superestimava ou subestimativa ao longo da linha de regressão.

Para modelos estatísticos em escala logarítmica, a discrepância logarítmica foi corrigida pelo Fator de Correção de Meyer (FM). No trabalho em questão, os modelos hipsométricos de Stoffels e Curtis utilizam o logaritmo natural, no entanto, em suas estimativas foram realizados tal procedimento pelo fator de correção de Meyer expresso pela Equação 4.

$$
F M=e^{0,5 * Q M_{\text {res }}}
$$

Em que: $e=$ base do logaritmo natural $(2,718281828 \ldots) ;$ e $Q M_{\text {res }}=$ quadrado médio dos resíduos.

\section{Teste de identidade}

Após o ajuste e a avaliação dos modelos, foi analisada a influência do espaçamento na relação hipsométrica por meio do teste de identidade de modelos proposto por Graybill (2000). Esse teste foi aplicado no modelo que apresentou resultados acurados para os dois tratamentos, o qual permitiu verificar a possibilidade de uma única equação representar a relação hipsométrica para os dados dos tratamentos agrupados.

O referido teste baseia-se na comparação entre a soma de quadrado dos resíduos em cada tratamento (modelo completo-MC) e a soma de quadrado da diferença para o modelo ajustado para os dados agrupados contendo todos os tratamentos (modelo reduzido-MR). Conforme Regazzi (1996), as hipóteses do teste são formuladas da seguinte forma:

- -Hipótese inicial $\left(\mathrm{H}_{0}\right)$ : Os modelos completos são estatisticamente iguais ao modelo reduzido; e

- -Hipótese alternativa $\left(\mathrm{H}_{1}\right)$ : nem todos os modelos completos são estatisticamente iguais ao modelo reduzido.

Assim, p-valor $\leq 0,05$ indica significância a 95\% de confiança e rejeição da hipótese $\mathrm{H}_{0}$, em que não é possível utilizar uma única equação para estimar a relação hipsométrica em diferentes espaçamentos, (REGAZZI;
SILVA, 2010; ARAÚJO et al., 2012; CAMPOS; LEITE, 2013). Todos os procedimentos de ajustes e análises estatísticas foram realizados no ambiente $R$ ( $R$ CORE TEAM, 2016).

\section{RESULTADOS E DISCUSSÃO}

Os parâmetros estimados e as estatísticas de precisão obtidos nos ajustes dos modelos hipsométricos estão na Tabela 3. Com base nos resultados do teste de significância dos modelos ajustados para altura total $(h)$, observa-se que as equações apresentaram ajustes significativos para os tratamentos, indicando que as mesmas podem explicar a variabilidade da altura total em função do diâmetro, conforme mencionado por Sanquetta et al. (2014).

Tabela 3. Parâmetros e estatísticas dos modelos de relação hipsométrica ajustados para altura total nos diferentes espaçamentos

\begin{tabular}{|c|c|c|c|c|c|c|}
\hline Trat. & Mod. & $\boldsymbol{\beta}_{0}$ & $\beta_{1}$ & $\boldsymbol{\beta}_{2}$ & $R^{2}$ aj. & $S_{y x}(\%)$ \\
\hline \multirow{6}{*}{$\mathrm{T} 1$} & 1 & $1,0207 *$ & $0,4806 *$ & & 0,465 & 10,37 \\
\hline & 2 & $2,7162^{*}$ & $-5,7026 *$ & & 0,436 & 10,65 \\
\hline & 3 & $11,6767^{*}$ & $-254,1254^{*}$ & & 0,322 & 11,67 \\
\hline & 4 & $-2,3640^{*}$ & $4,6838^{*}$ & & 0,457 & 10,44 \\
\hline & 5 & $14,086^{*}$ & $-54,475^{*}$ & & 0,408 & 10,91 \\
\hline & 6 & $16,1852^{*}$ & $2,3006^{*}$ & $0,09158 *$ & 0,463 & 10,39 \\
\hline \multirow{6}{*}{$\mathrm{T} 2$} & 1 & 0,5970* & $0,6777^{*}$ & & 0,570 & 12,82 \\
\hline & 2 & $3,0949 *$ & $-9,5695^{*}$ & & 0,558 & 13,00 \\
\hline & 3 & $15,1686^{*}$ & $-660,6275^{*}$ & & 0,468 & 14,26 \\
\hline & 4 & $-9,5408^{*}$ & $7,8257^{*}$ & & 0,566 & 12,87 \\
\hline & 5 & $19,1770 *$ & $-108,4869 *$ & & 0,532 & 13,37 \\
\hline & 6 & $18,7612^{*}$ & $4,0412 *$ & $0,1236^{*}$ & 0,571 & 12,79 \\
\hline
\end{tabular}

* significativo $(\mathrm{p} \leq 0,05) ; \beta=$ coeficientes do modelo; $\mathrm{R}^{2}$ aj. = coeficiente de determinação ajustado; e Syx $(\%)$ = erro padrão da estimativa em percentagem.

As equações ajustadas para os diferentes espaçamentos apresentaram coeficientes de determinação ajustado ( $R^{2}$ aj.) variando de 0,32 a 0,57 e erro padrão médio em percentagem (Syx\%) de 10,3\% a $14,2 \%$. A relação hipsométrica em ambos os tratamentos apresentou uma relação biológica fraca, em que há baixa correlação entre a altura e o diâmetro dos povoamentos, evidenciada pelos baixos valores de coeficiente de determinação ajustado ( $R^{2} a j$.).

No entanto, os erros padrões da estimativa nos melhores ajustes podem ser considerados satisfatórios. Resultados semelhantes foram observados por Nicoletti et al. (2016), em estudo da relação hipsométrica para Pinus taeda L. em diferentes fases do ciclo de corte em 
Santa Catarina, e por Rodrigues et al. (2017), estudando a relação hipsométrica de Pinus taeda no estado do Paraná.

Os baixos valores do coeficiente de determinação ajustado ( $R^{2}$ aj.) podem ser ocasionados pela descaracterização da relação hipsométrica, em que as alturas tendem a se homogeneizar, independente da distribuição diamétrica, conforme mencionado por Machado et al. (2011). Pelissari et al. (2014) acrescentam que os valores de $\left(R^{2}\right.$ aj.) reduzem à medida que aumenta a idade do povoamento, sendo encontrados valores baixos em plantios que mais desenvolvidos.

Apesar das análises dos coeficientes e das estatísticas de precisão fornecerem importantes subsídios para avaliação da acurácia dos modelos, não garantem isoladamente inferir sobre o desempenho deles, uma vez que tratam apenas das médias das variações das estimativas totais, subsidiando apenas como um indicativo da qualidade do ajuste (SOUZA et al., 2016). Dessa forma, pode ser visualizado a distribuição dos resíduos das equações hipsométricas para estimativa da altura total nos diferentes espaçamentos (Figura 2).

A distribuição gráfica dos resíduos das equações mais acuradas (Logística e Stoffels) para estimativa da $h$ nos diferentes tratamentos apresentou-se dispersos adequadamente em torno da média. Porém, observou-se tendências em superestimar ou subestimar a variável $h$ em função da variável $d$ nos ajustes das demais equações.

As curvas estimadas apresentaram comportamento esperado para tal relação, com tendência estabilização do crescimento em altura com acréscimo dos diâmetros (Figura 3). Nota-se que a curva média estimada pelo modelo de Stoffels não representou de forma acurada os dados observados para o tratamento T1. O modelo Logístico representou adequadamente os dados observados em ambos os tratamentos, confirmando sua acurácia para estimativa da altura dos povoamentos estudados.
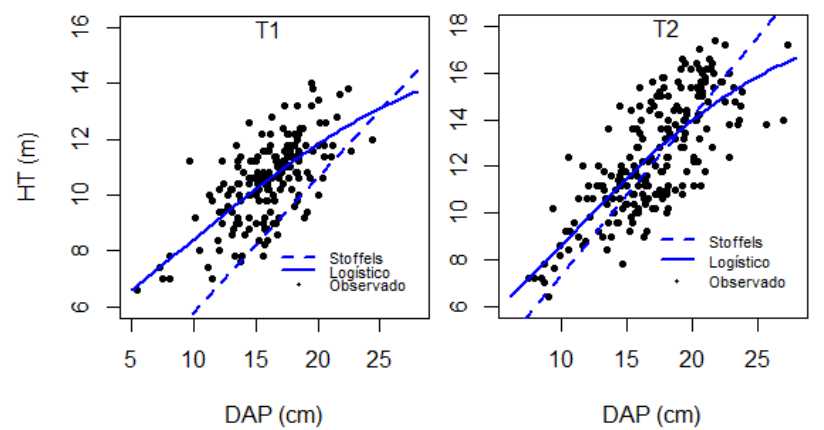

Figura 3. Curvas hipsométrica do modelo logística e Stoffels para os tratamentos estudados.
Com base nas estatísticas de ajuste, análise gráfica de resíduos e curva média versus dados observados, o modelo logístico proporciona estimativas mais acuradas para predição da altura total nos diferentes tratamentos.

\section{Teste de identidade}

Como um mesmo modelo apresentou resultados satisfatórios para os dois tratamentos, surge a necessidade de avaliar a possibilidade da utilização de uma mesma equação ajustada para as alturas das árvores nos diferentes espaçamentos. Para isso, adotou-se o teste de identidade de modelos, cujos resultados estão apresentados na Tabela 4.

Tabela 4. Análise de variância para o teste de identidade de Graybill

\begin{tabular}{ccccccc}
\hline FV & GL & SQ & QM & $\mathbf{F}_{\text {calculado }}$ & $F_{\text {tabelado }}$ & p-valor \\
\hline MC & 6 & $55.592,28$ & & & & \\
MR & 3 & $55.350,75$ & & & & \\
Redução & 3 & 241,53 & 80,51 & 41,62 & 2,627 & 0,000 \\
Resíduo & 399 & 771,84 & 1,93 & & & \\
\hline Total & $\mathbf{4 0 5}$ & $\mathbf{5 6 3 6 4 , 1}$ & & & & \\
\hline
\end{tabular}

$\mathrm{FV}=$ fonte de variação; $\mathrm{GL}$ = grau de liberdade; $\mathrm{SQ}=$ soma dos quadrados; $\mathrm{QM}=$ quadrado médio; $\mathrm{MC}=$ modelo completo; $\mathrm{e}$ $\mathrm{MR}=$ modelo reduzido.

O teste de identidade de modelos foi realizado para os dois tratamentos agrupados (T1 e T2) contendo as alturas totais, apresentando resultados significativos ao nível de 95\% de significância ( $p \leq 0,05$ ) e rejeição da hipótese de que não há diferença significativa entre as curvas estimada. Assim, as equações diferem entre si e a soma de quadrado de resíduos para os diferentes tratamentos não é estatisticamente igual à soma de quadrados do resíduo do modelo comum ( $\mathrm{MC})$, não sendo recomendado para a estimativa da altura do povoamento abordado em diferentes densidades.

Com isso, é possível evidenciar que o espaçamento influenciou de forma significativa a relação hipsométrica. Resultados semelhantes também foram observados por Araújo et al. (2012), testando modelos hipsométricos para Candeia (Eremanthus erythropappus (DC.) MacLeish) em diferentes espaçamentos de plantio em Minas Gerais, os quais concluíram que não é possível aplicar uma única equação contemplando diferentes espaçamentos. Nicoletti et al. (2016), em estudo da relação hipsométrica para Pinus taeda em Santa Catarina, concluíram que é necessário equações específicas para diferentes fases do ciclo de corte. 
Tratamento 1
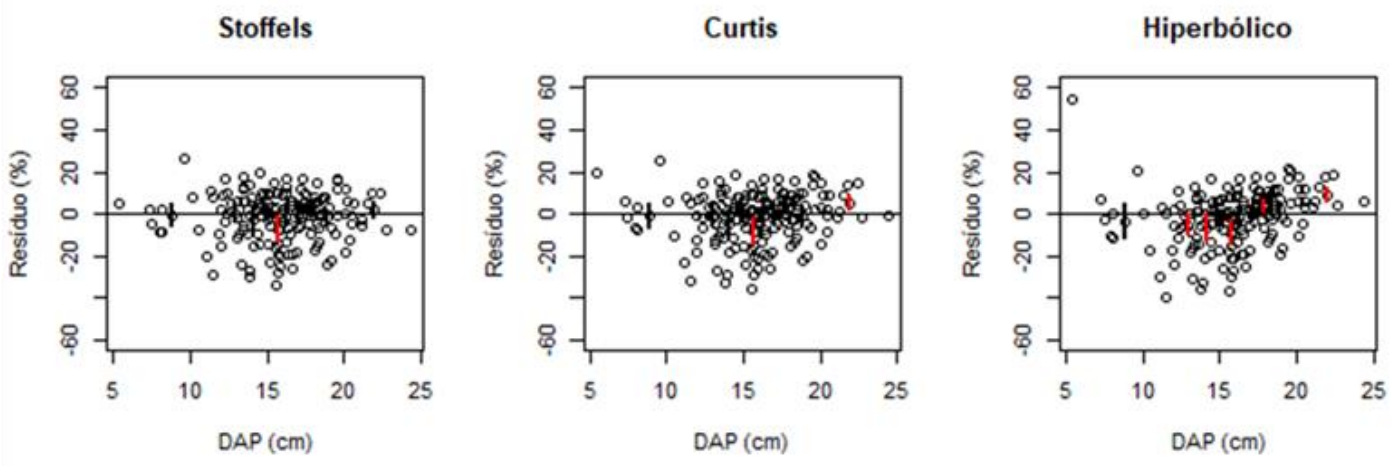

Henriksen

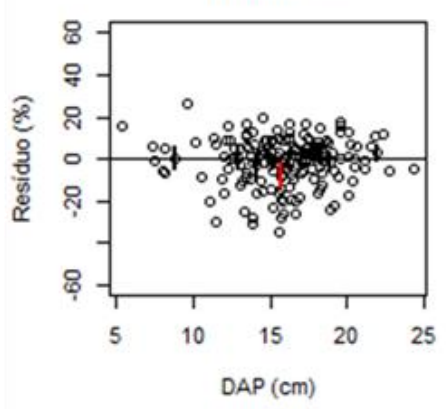

Tratamento 2
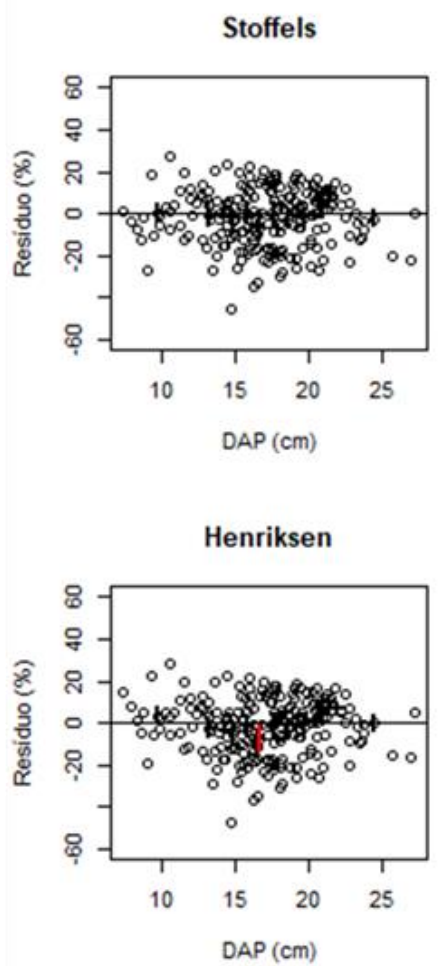

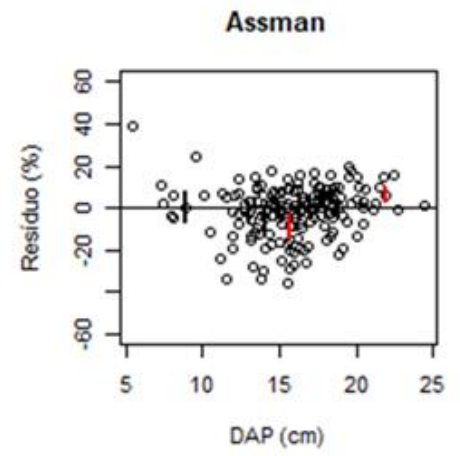

Curtis
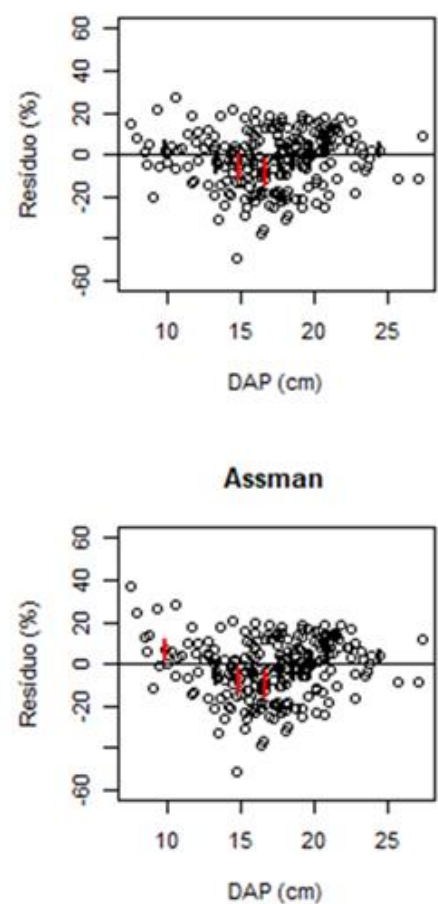

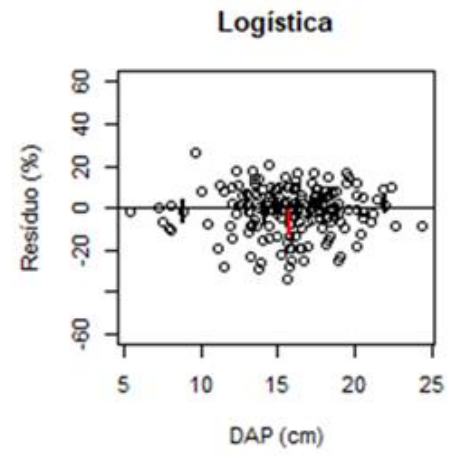

Hiperbólico

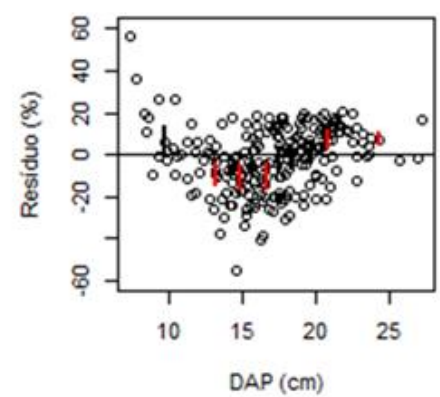

Logistica

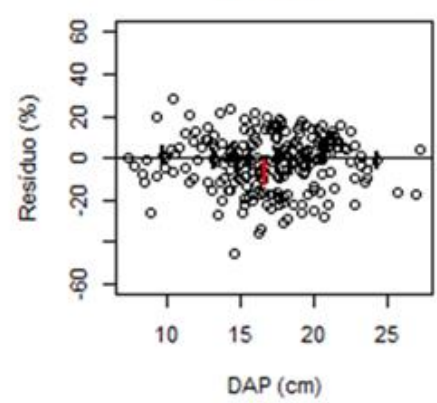

Figura 2. Distribuição dos resíduos nos ajustes dos modelos para os tratamentos T1 e T2.

\section{CONCLUSÕES}

Dentre as equações ajustadas para estimativa da altura de Pinus caribaea var. hondurensis nos diferentes espaçamentos, o modelo não linear logístico apresenta maior acurácia de ajuste.
O espaçamento influência de forma significativa a relação hipsométrica de Pinus caribaea var. hondurensis aos 8 anos de idade, em que uma única equação não deve ser usada para representar a altura total. 


\section{REFERÊNCIAS}

ALVARES, C. A.; STAPE, J. L.; SENTELHAS, P. C.; GONÇALVES, J. L. M.; SPAROVEK, G. Köppen's climate classification map for Brazil. Meteorologische Zeitschrift, v. 22, n. 6, p. 711-728, 2013.

ARAÚJO, E. J. G.; PELISSARI, A. L.; DAVID, H. C.; SCOLFORO, J. R.; NETTO, S. P.; MORAIS, V. A. Relação hipsométrica para candeia (Eremanthus erythropappus) com diferentes espaçamentos de plantio em Minas Gerais, Brasil. Pesquisa Florestal Brasileira, v. 32, n. 71, p. 257-268, 2012.

BARTOSZECK, A. C. P. S.; MACHADO, S. A.; FIGUEIREDO FILHO, A.; OLIVEIRA, E. B. Modelagem da relação hipsométrica para bracatingais da região metropolitana de Curitiba-PR. Floresta, v. 32 , n. 2, p. 189-204, 2002.

CAMPOS, J. C. C.; LEITE, H. G. Mensuração florestal: perguntas e respostas. 4. ed. Viçosa: UFV, 2013. 605 p.

CURTIS, R. O. Height diamenter and height diamenter age equations for second growth Douglas-fir. Forest Science, v. 13, n. 4, p. 356-375, 1967.

DONADONI, A. X.; PELISSARI, A. L.; DRESCHER, R.; ROSA, G. D. da. Relação hipsométrica para Pinus caribaea var.hondurensis e Pinus tecunumanii em povoamento homogêneo no Estado de Rondônia. Ciência Rural, v. 40, n. 12, p. 2499-2504, 2010.

GRAYBILL, F. A. Theory and application of the linear model. Belmont: Duxbury, 2000. $704 \mathrm{p}$

IBÁ - Indústria brasileira de árvores. Anuário IBÁ 2017: ano base 2016. Brasília: 2017. 100 p.

MACHADO, S. A.; BARROS, D. A.; SCOLFORO, J. R.; ACERBI JÚNIOR, F. W. The effects of successive thinning on the hypsometric function for Pinus oocarpa stands. Floresta, v. 41, n. 2, p. 397-406, 2011.

MEHTATALO, L. Forest Biometrics with examples in R: lecture notes for the forest biometrics course. University of Helsinki, 2008. $186 \mathrm{p}$.

MELO, L. C.; BARRETO, P. A. B.; OLIVEIRA, F. G. R. B.; NOVAES, A. B. Estimativas volumétricas em povoamento de Pinus caribaea var. hondurensis no Sudoeste da Bahia. Pesquisa Florestal Brasileira, v. 33, p. 381-388, 2013.

NICOLETTI, M. F.; SOUZA, K.; SILVESTRE, R.; FRANCA, M. C.; ROLIM, F. A. Relação hipsométrica para Pinus taeda L. em diferentes fases do ciclo de corte. FLORAM, v. 1, p. 80-89, 2016.

PELISSARI, A. L.; LANSSANOVA, L. R.; DRESCHER, R. Modelos volumétricos para pinus tropicais, em povoamentos homogêneo, no Estado de Rondônia. Pesquisa Florestal Brasileira, v. 31, n. 67, p. 173-181, 2011.

PELISSARI, A. L.; FIGUEIREDO FILHO, A.; CALDEIRA, S. F.; MACHADO, S. do A. Geostatistic applied to spatial modeling of hypsometric relationships in forest stands. American International Journal of Contemporary Research, v. 4, n. 7, p. 67-76, 2014.
R CORE TEAM. R: a language and environment for statistical computing. Vienna: R Foundation for Statistical Computing, 2016.

REGAZZI, A. J.; SILVA, C. H. O. Testes para verificar a igualdade de parâmetros e a identidade de modelos de regressão não-linear em dados de experimento com delineamento em blocos casualizados. Revista Ceres, v. 57, n. 3, p. 315-320, 2010.

REGAZZI, A. J. Teste para verificar a identidade de modelos de regressão. Pesquisa Agropecuária Brasileira, v. 31, n. 1, p. 1-17, 1996.

RODRIGUES, C. K.; LOPES, E. S.; FILHO, A. F.; PELISSARI, A. L. Identidade de modelos hipsométricos e volumétricos de povoamentos de Pinus com diferentes idades submetidos ao desbaste mecanizado. BIOFIX Scientific Journal, v. 2, p. 54-59, 2017.

RAMALHO, A. R.; GODINHO, V. P.; UTUMI, M. M.; JESUS, J. Condicionantes agroclimáticas para a ricinocultura no sul de Rondônia. In: CONGRESSO BRASILEIRO DE MAMONA, 1., 2004, Campina Grande. Anais... Campina Grande: Embrapa Algodão, 2004.

SANQUETTA, C.R, BEHLING, A. CORTE, A. P. D. RUZA, M. S. SIMON, A. A, SÃO JOSÉ, J. F. B. Relação hipsométrica em inventário pré-corte em povoamentos de Acacia mearnsii De Wild. Científica, v. 42, n. 1, p. 80-90, 2014.

SCOLFORO, J. R. S. Biometria florestal. Lavras: UFLA/FAEPE, 2005. $352 \mathrm{p}$.

SHIMIZU, J. Y. Pinus na silvicultura brasileira. Revista da madeira, v. 16, n. 99, p. 4-14, 2006.

SOUZA, R. R.; GILCIANO, N. S.; MURTA JÚNIOR, L. S.; PELLI, E.; OLIVEIRA, M. L. R.; ABRAHAO, C. P.; LEITE, H. G. Forma de fuste de árvores de eucalipto em plantios adensados. Scientia Forestalis, v. 44, p. 109-40, 2016.

VENDRUSCOLO, D. G. S.; CHAVES, A. G. S.; SILVA, R. S.; SOUZA, H. S.; MEDEIROS, R. A.; MOTTA, A. S.; SILVA, F. T. Identidade em modelos hipsométricos para Tectona grandis com diferentes espaçamentos em Cáceres-MT. Nativa, v. 3, p. 44-49, 2015. 